\title{
Verbal Fluency Test in Children with Neurodevelopmental Disorders
}

\author{
Esteban Vaucheret Paz ${ }^{1}$ Celeste Puga ${ }^{1}$ Christy Ekonen ${ }^{1}$ Paula Pintos ${ }^{1}$ Isabel Lascombes ${ }^{1}$ \\ Soledad De Vita ${ }^{1}$ Mariana Leist ${ }^{1}$ Mariela Corleto ${ }^{1}$ María José García Basalo ${ }^{1}$ \\ ${ }^{1}$ Department of Child Neurology, Hospital Italiano de Buenos Aires, \\ Buenos Aires, Argentina \\ Address for correspondence Esteban Vaucheret Paz, Department \\ of Child Neurology, Hospital Italiano de Buenos Aires, Buenos Aires, \\ Argentina (e-mail: esteban.vaucheret@hiba.org.ar).
}

J Neurosci Rural Pract 2020;11:95-99

\begin{abstract}
Keywords

- neuropsychological test

- verbal fluency test

- language disorders

- executive function

Background The verbal fluency task is a widely used psychometric test to account for cognitive functions, particularly, verbal and executive functions. Being an easy and fast test to administer, it is a good neuropsychological tool in low technology environments. Our objective was to analyze the performance in verbal fluency of Spanish-speaking children with neurodevelopmental disorders.

Methods We performed a retrospective cross-sectional study to analyze the performance of children who had undergone a verbal fluency test in a neuropsychological assessment.

Results We included 115 participants. There were 41 (35.65\%) participants with low intellectual performance (LIP), 63 (54.78\%) with attention deficit hyperactive disorder (ADHD), and 11(9.57\%) participants with dyslexia. Participants with LIP showed lower phonological and semantic fluency scores than participants with ADHD, and a lower performance in semantic fluency than the dyslexia group. The probability of having LIP was 6.12 times greater when somebody had a scale score lower than 7 in the phonological task and it was 7.9 times greater when the scale score was lower than 7 in the semantic task.

Conclusion There was a direct relationship between Full Scale Intelligence Quotient and verbal fluency test performance, the latter being a brief and effective neuropsychological test that can reveal deficit not only in executive functions and verbal abilities but also detect LIP.
\end{abstract}

\section{Introduction}

As human beings we have an individual and internalized knowledge of the vocabulary of our language called lexicon. We can access it when we want to represent words for a specific action, situation, or object. This access to the object's name depends on phonological skills and executive functions. There is a relationship between learning vocabulary and categorizing it in the lexicon, because categorization requires the existence of a mental representation of meaning, which is mapped to form lexical items. ${ }^{1}$

The verbal fluency test can provide information about the storage capacity, the ability to retrieve information and evaluates strategies used to search for words.
The verbal fluency test is a short psychometric test, which is widely included in almost every neuropsychological assessment because it is sensitive to cognitive impairment due to a variety of etiologies. ${ }^{2,3}$ It typically consists of two tasks: semantic category (or semantic fluency [SF]) and letter fluency (or phonological fluency [PF]). In the standard version of the test, in the first task participants are given 1 minute to generate as many words as possible within a semantic category. For example, they are asked to say words from a given category that could be "animals," "food," "clothing," or "tools." In the PF task, participants are asked to say words that start with a given letter. The sequence of letters usually used is " $F$," "A," "S," (FAS) but in children the number of categories or letters offered may 
be reduced to two. In Spanish-speaking countries, the use of the letters "P" and "M" has been proposed. ${ }^{4}$

The widespread use of verbal fluency tests is probably due to the utility of these tests for measuring both verbal ability and executive control. The validity of the fluency test as a tool to assess verbal ability has been confirmed in several studies. Lexical access ability is the ability to retrieve the grammatical representations and sound forms of words from the mental lexicon. ${ }^{5}$ That is, those who have less vocabulary will produce fewer words than those with larger vocabulary. Children with specific language impairment or with dyslexia show a lower performance in these tests compared with typically developing children. ${ }^{6}$

The validity of the fluency test as a tool to assess executive function is also well documented. Executive function is a set of functions that regulate thinking and behavior to achieve a specific goal. There is enough evidence in the scientific literature pointing to lower performance in verbal fluency tests in both, children with attention deficit hyperactive disorder (ADHD), ${ }^{7}$ and in patients with frontal lobe lesion. ${ }^{8}$ Because of this, the administration of verbal fluency tests has been proposed for screening of cognitive deficiency in patients with impairment in executive functions, such as Parkinson's, ${ }^{9}$ multiple sclerosis, ${ }^{10}$ or dementia. ${ }^{11}$

Consistent with clinical observations, neuroimaging studies on healthy people have revealed the involvement of overlapping, but not the same, brain circuits in both tasks. SF was found to be associated with activation in ventral-anterior left inferior frontal gyrus, whereas PF was found to be represented in the posterior-dorsal left frontal gyrus ${ }^{12,13}$ as well as in presupplementary motor area and left caudate. ${ }^{14}$ In summary, both clinical and neuroimaging evidence suggests that verbal ability may be better reflected in the semantic task, rather than in the PF task, whereas executive ability may be more strongly reflected in the PF task.

Although extensive assessment of verbal ability and executive functions is important to obtain an accurate description of a patient's neuropsychological profile, such an evaluation is not always available in clinical practice. Therefore, the existence of a brief screening tool, which is easy to administer and also detects verbal and executive deficits in patients, would be of great value for clinicians working with them.

Despite the existence of some studies about the performance in pediatric population on verbal fluency tasks, this test has not been as carefully studied as in adulthood. Therefore, the objective of the present study was to analyze the performance of children with neurodevelopmental disorders in the verbal fluency test as a neuropsychological tool of easy, brief, and low technology administration.

\section{Materials and Methods}

\section{Design}

We performed a retrospective cross-sectional study.

\section{Population}

Subjects between 6 and 17 years of age who underwent a neuropsychological assessment available were included.
Once the participants were selected, a neuropediatrician validated the diagnosis according to the DSM-5 (Diagnostic and Statistical Manual of Mental Disorders, 5th Edition) criteria by means of the medical records, the neurological examination, and an interview with the parents.

Children with Full Scale Intelligence Quotient (FSIQ) $<80$ were classified in the group called low intellectual performance (LIP).

\section{Exclusion Criteria}

Participants with diagnosis of developmental disorder associated with epilepsy, schizophrenia, Tourette syndrome, central nervous system tumors, or malformations were excluded.

Children with a FSIQ below 50 points were excluded to avoid compromising the correct understanding of the instructions.

Participants who did not complete (all) the neuropsychological assessments were also excluded.

\section{Materials}

Participants with validated diagnosis underwent a neuropsychological assessment conducted by a neuropsychologist. The assessment included an intelligence test (Wechsler Intelligence Scale for Children, version WISC V in Spanish), the verbal fluency tests of the-NEPSY II (Developmental Neuropsychological Assessment, II Edition, Spanish version) neuropsychological battery (verbal fluency subtest with semantic and phonological tasks). ${ }^{15}$

The following primary indexes were obtained on the basis of the intelligence test: Verbal Comprehension Index, Fluid Reasoning Index, Visuospatial Index, Working Memory Index, Processing Speed Index, and FSIQ ${ }^{16}$; a standard score with an average of 100 and a standard deviation (SD) of 15 was applied for the analysis.

The verbal fluency test, from the NEPSY II neuropsychological battery, consists of two subtests: PF and SF. The PF subtest comprised two 1-minute tests where the participants were required to say as many words as possible starting with the letters "M" in the first case, and with the letter "P" in the other. In the SF task, participants were given 1 minute to say names of animals and then another minute to say names of food and beverages. Scale scores were used for the analysis of these two tests, considering 10 as average and a SD of 3.

\section{Analysis}

The continuous variables assessed were age, FSIQ and the primary score indexes from the Wechsler scale, verbal fluency subtest, and overall sentiment analysis from each video. The categorical variables were sex and medical diagnosis. The relationship between PF task and SF task with the IQ was analyzed using chi-squared test, considering PF and SF as dichotomous variables using a scale score of 7 as cutoff value. To analyze the association between LIP and fluency, we used a logistic regression model adjusted by sex and age.

The normal distribution of the continuous variables was assessed by means of the Shapiro-Wilk test and graphically, by using a histogram. 
The continuous variables were expressed as mean and SD if the distribution was normal, and median and interquartile range if the distribution was asymmetrical. Categorical variables were expressed in percentage or ratios.

For the comparison of normal continuous variables, a $t$-test was used for paired samples and, if assumptions of normal distribution were not met, the Wilcoxon signed-rank test was used.

For categorical variables, the chi-squared test or the Fisher's exact test was used if the value expected in any of the cells was above or below 5 , respectively.

A linear regression was used considering the overall sentiment analysis as a dependent variable and age, FSIQ and Wechsler scale indexes as independent variables. The analysis was conducted using the statistical package Stata 13.0.

The statistical difference was considered at $p<0.05$.

The study conducted has complied with the ethical standards set forth in the 1975 Declaration of Helsinki amended in $2005 .{ }^{17}$

\section{Results}

We included 119 subjects, excluding 4 of them because they did not complete all the neuropsychological tests required. The study was finally conducted on 115 participants with a mean age of 10.6 years ( $S D \pm 2.83$ ). There were 80 males (69.56\%). The mean scale score in the SF test was 7.44 (SD \pm 3.36$)$ and the phonological verbal fluency test's mean scale score $(P F)$ was $5.70(S D \pm 2.57)$. FSIQ mean was 78.7 (SD \pm 16.5).

The characteristics of the participants according to the diagnostic category such as LIP, ADHD, and dyslexia are described in - Table 1. There were no differences in relation to age and sex between the three groups. Participants with LIP showed lower PF than ADHD group. As for SF, differences were observed between participants with LIP and ADHD and also between LIP and dyslexia groups.

Taking into account the diagnoses, the population was distributed as follows: 41 (35.65\%) participants showed LIP, 63 (54.78\%) patients had an ADHD, and 11 (9.57\%) participants had a diagnosis of dyslexia. - Table 2 shows the proportion of participants with low performance in PF and SF task according to diagnosis.

When logistic regression was performed, we observed that the probability of having LIP when somebody had had a scale score lower than 7 in the PF task was 6.12 times greater (odds ratio $[\mathrm{OR}]=6.12, p<0.001 ; 95 \%$ confidence interval $[\mathrm{CI}]$ $=2.16-17.33)$. On the other hand, we performed the logistic regression between the LIP group and the one that obtained a lower performance in the SF task; we observed that the probability of having LIP when achieving a scale score lower than 7 in the SF task was 7.9 times greater $(\mathrm{OR}=7.89, p<0.001$; $95 \% \mathrm{CI}=3.32-18.76)$. The effect remained after adjusting for sex and age ( - Table 3 ).

\section{Discussion}

The verbal fluency test is a widely used psychometric tool in neuropsychological assessment, because it has the ability to detect dysfunction in verbal skills and executive functions. The performance will be affected differently, because the SF is usually affected by verbal skills, whereas the PF is affected by the executive functions. ${ }^{18}$ These findings are independent of sex, ${ }^{19}$ ethnic or cultural aspects. ${ }^{20}$

The different performance observed in PF and SF suggests that there could be different retrieval mechanisms employed in each task. For example, the retrieval of a word (e.g., potato) will activate semantically associated words (e.g., tomato, meat, and egg) ${ }^{21}$; so, the SF task resembles everyday production and participants can exploit existing links between

Table 1 Characteristics of 115 participants by diagnostic category

\begin{tabular}{|l|l|l|l|l|}
\hline & LIP $(\boldsymbol{n}=\mathbf{4 1})$ & ADHD $(\boldsymbol{n}=63)$ & Dyslexia $(\boldsymbol{n}=\mathbf{1 1})$ & $\boldsymbol{p}$-Value \\
\hline Age (years) (SD) & $10.8(8.9-13.4)$ & $10(8.11-12.4)$ & $10.3(8-11.1)$ & 0.29 \\
\hline Gender, male, $\mathrm{n}(\%)$ & $31(76)$ & $42(67)$ & $7(63)$ & 0.57 \\
\hline Semantic fluency median (95\% Cl) & $4(3-7)$ & $9(6-11)$ & $8(7-11)$ & $0.0001^{\text {a }}$ \\
\hline Phonological fluency, median (95\% Cl) & $4(3-6)$ & $6(4-8)$ & $6(4-8)$ & $0.0005^{\text {b }}$ \\
\hline VIQ, median (95\% Cl) & $67(59-79)$ & $93(87-99)$ & $91(87-98)$ & $0.0001^{\text {a }}$ \\
\hline FSIQ, median (95\% Cl) & $63(54-71)$ & $88(80-96)$ & $83(80-98)$ & $0.0001^{\text {a }}$ \\
\hline
\end{tabular}

Abbreviations: ADHD, attention deficit hyperactive disorder; Cl, confidence interval; FSIQ, Full Scale Intelligence Quotient; LIP, low intellectual performance; SD, standard deviation; VIQ, Verbal Intelligence Quotient.

aThe differences were statistically significant between LIP patients and the other 2 groups.

${ }^{\text {b} T h e ~ d i f f e r e n c e s ~ w e r e ~ s t a t i s t i c a l l y ~ s i g n i f i c a n t ~ b e t w e e n ~ t h e ~ L I P ~ g r o u p ~ a n d ~ t h e ~ g r o u p ~ o f ~ p a t i e n t s ~ w i t h ~ A D H D . ~}$

cFor the analysis of semantic fluency and phonological fluency test, we used scale scores, whereas for the VIQ and FSIQ, we used standard score.

Table 2 Proportion of participants with phonological fluency and semantics fluency test according to diagnosis

\begin{tabular}{|l|l|l|l|}
\hline & \multicolumn{1}{|c|}{ LIP $(\boldsymbol{n}=\mathbf{4 1})$} & \multicolumn{1}{|c|}{ ADHD $(\boldsymbol{n}=\mathbf{6 3})$} & \multicolumn{1}{c|}{ Dyslexia $(\boldsymbol{n}=11)$} \\
\hline Low phonological fluency test, $n(\%)$ & $36(88)$ & $32(51)$ & $8(73)$ \\
\hline Low semantic fluency test, $n(\%)$ & $30(73)$ & $17(27)$ & $2(18)$ \\
\hline
\end{tabular}

Abbreviations: ADHD, attention deficit hyperactive disorder; LIP, low intellectual performance. 
Table 3 Independent predictors of low semantic and phonologic fluency among 115 participants

\begin{tabular}{|c|c|c|c|c|}
\hline \multicolumn{5}{|c|}{ Independent predictors of low semantic fluency } \\
\hline & \multicolumn{2}{|c|}{ Univariate OR } & \multicolumn{2}{|c|}{ Multivariate OR } \\
\hline & OR $(95 \% \mathrm{Cl})$ & $p$-Value & OR $(95 \% \mathrm{Cl})$ & $p$-Value \\
\hline LIP (FSIQ < 80) & $7.89(3.32-18.76)$ & $<0.001^{\mathrm{a}}$ & $7.56(3.14-18.17)$ & $<0.001^{\mathrm{a}}$ \\
\hline Age (years) & $1.11(0.97-1.27)$ & 0.109 & $1.07(0.92-1.24)$ & 0.34 \\
\hline Sex (male) & $1.16(0.52-2.61)$ & 0.708 & $0.95(0.38-2.37)$ & 0.92 \\
\hline \multicolumn{5}{|c|}{ Independent predictors of low phonological fluency } \\
\hline & \multicolumn{2}{|c|}{ Univariate OR } & \multicolumn{2}{|c|}{ Multivariate OR } \\
\hline & OR $(95 \% \mathrm{Cl})$ & $p$-Value & OR $(95 \% \mathrm{Cl})$ & $p$-Value \\
\hline LIP $(\mathrm{FSIQ}<80)$ & $6.12(2.16-17.33)$ & $0.001^{\mathrm{a}}$ & $6.15(2.11-17.92)$ & $0.001^{\mathrm{a}}$ \\
\hline Age (years) & $1(0.87-1.15)$ & 0.12 & $0.96(0.82-1.12)$ & 0.65 \\
\hline Sex (male) & $2.48(1.09-5.68)$ & 0.03 & $2.34(0.97-5.66)$ & 0.057 \\
\hline
\end{tabular}

Abbreviations: ADHD, attention deficit hyperactive disorder; Cl, confidence interval; FSIQ, Full Scale Intelligence Quotient; LIP, low intellectual performance; OR, odds ratio; VIQ, Verbal Intelligence Quotient.

aThe differences were statistically significant.

related concepts and retrieved response. By contrast, the links between words beginning with the same letter may be less accessible so that participants must suppress the activation of semantically or associatively related words and implement novel search strategies to carry out the PF task. ${ }^{22}$ In addition, the PF task may engage cognitive skills (i.e., executive functions) whose deficits are also reflected in fluid intelligence results. $^{23}$

There have been studies documenting a decrease in PF production in participants with language and reading disorders who were given the test with the letters "F," "A," and "S." 24 According to this, in our study using the letters "P" and "M," the $73 \%$ of the participants with dyslexia failed in the PF, whereas only $18 \%$ failed in the SF task.

On the other hand, a lower performance has been reported in the PF task in participants with ADHD with the letters "F," "A," and "S." Those results were not associated with age, sex, and educational level attained. ${ }^{15}$ In agreement with scientific literature, we found that in our population $51 \%$ of the ADHD patients failed in the PF task.

Finally, the present study demonstrates that there is a direct relationship between FSIQ and the performance obtained in the verbal fluency test in our population, because those participants who obtained a PF score lower than 7 were six times more likely to have a LIP and those who obtained a low performance in the SF task were nearly eight times more likely to have a LIP than those who achieved a typical performance on those test.

The present study has some limitations that should be taken into account in future investigations. The population in this study consisted in participants who had undergone a neuropsychological assessment because they had been referred to the Child Neurology Department. Therefore, the results could be different to those in a typically developing population.

Nevertheless, and beyond these limitations, we believe that our results have an important clinical implication because the access and the necessary time for an extensive neuropsychological assessment are not always available. We propose that the verbal fluency task can be a brief and effective neuropsychological tool to account for cognitive functions, not only for verbal and executive functions but also for detecting LIP.

\section{Conclusion}

Although no single test can replace the value of a complete neuropsychological assessment, our study reveals that the verbal fluency test could be used as a brief, reliable, and easy to administer tool to assess children with neurodevelopmental disorder. Those patients with poor performance on this test should undergo a complete neuropsychological assessment to clarify their diagnosis.

\section{Note}

This is a retrospective cross-sectional study.

\section{Authors' Contributions}

All the authors have participated in the conception and the design of the research. Christy Ekonen, Paula Pintos, Isabel Lascombes, Soledad De Vita, Mariela Corleto, María José García Basalo collected the data. The statistical analysis and interpretation of the results was performed by Esteban Vaucheret Paz, Celeste Puga, and Mariana Leist. The writing of the research was done by Esteban Vaucheret Paz and Celeste Puga. All the authors participated in the final correction and agreed with the final manuscript.

\section{Ethical Approval}

The authors confirm that the research was conducted according to the principles of the Helsinki Declaration and that all mandatory laboratory health and safety procedures have been complied.

\section{Funding}

This work was supported by the authors.

Conflict of Interest

None declared. 


\section{References}

1 dos Santos IMM, Chiossi JSC, Soares AD, de Oliveira LN, Chiari BM. Phonological and semantic verbal fluency: a comparative study in hearing-impaired and normal-hearing people. CoDAS 2016;26(6):434-438

2 Ruff RM, Light RH, Parker SB, Levin HS. The psychological construct of word fluency. Brain Lang 1997;57(3):394-405

3 Kavé G. Phonemic fluency, semantic fluency, and difference scores: normative data for adult Hebrew speakers. J Clin Exp Neuropsychol 2005;27(6):690-699

4 Butman J, Allegri RF, Harris P, Drake M. [Spanish verbal fluency. Normative data in Argentina]. Medicina (B Aires) 2000;60(5 Pt 1) :561-564

5 Levelt WJ, Roelofs A, Meyer AS. A theory of lexical access in speech production. Behav Brain Sci [Internet]. 1999;22(1):1-38-75

6 Weckerly J, Wulfeck B, Reilly J. Verbal fluency deficits in children with specific language impairment: slow rapid naming or slow to name? Child Neuropsychol 2001;7(3):142-152

7 Takács Á, Kóbor A, Tárnok Z, Csépe V. Verbal fluency in children with ADHD: strategy using and temporal properties. Child Neuropsychol 2014;20(4):415-429

8 Schwartz S, Baldo J. Distinct patterns of word retrieval in right and left frontal lobe patients: a multidimensional perspective. Neuropsychologia 2001;39(11):1209-1217

9 Torralva T, Laffaye T, Báez S, et al. Verbal fluency as a rapid screening test for cognitive impairment in early Parkinson's disease. J Neuropsychiatry Clin Neurosci 2015;27(3):244-247

10 Connick P, Kolappan M, Bak TH, Chandran S. Verbal fluency as a rapid screening test for cognitive impairment in progressive multiple sclerosis. J Neurol Neurosurg Psychiatry 2012;83(3):346-347

11 Olazarán J, Hoyos-Alonso MC, del Ser T, et al. Practical application of brief cognitive tests. Neurol (Barcelona, Spain) [Internet]. 2016 Apr [cited 2016 Sep 8];31(3):183-94

12 Katzev M, Tüscher O, Hennig J, Weiller C, Kaller CP. Revisiting the functional specialization of left inferior frontal gyrus in phonological and semantic fluency: the crucial role of task demands and individual ability. J Neurosci 2013;33 (18):7837-7845
13 Costafreda SG, Fu CH, Lee L, Everitt B, Brammer MJ, David AS. A systematic review and quantitative appraisal of fMRI studies of verbal fluency: role of the left inferior frontal gyrus. Hum Brain Mapp 2006;27(10):799-810

14 Grogan A, Green DW, Ali N, Crinion JT, Price CJ. Structural correlates of semantic and phonemic fluency ability in first and second languages. Cereb Cortex 2009;19(11):2690-2698

15 Abreu N, Argollo N, Oliveira F, Cardoso AL, Bueno JLO, Xavier G. Semantic and phonologic verbal fluency tests for adolescents with ADHD. Clin Neuropsychiatry 2013;10(2):63-71

16 Na SD, Burns TG. Wechsler Intelligence Scale for Children-V: test review. Appl Neuropsychol Child 2016;5(2):156-160

17 Declaración universal sobre Bioética y Derechos Humanos: UNESCO [Internet]. [cited 2017 Sep 19]. Available at: http:// portal.unesco.org/es/ev.php-URL_ID=31058\&URL_DO=DO_ TOPIC\&URL_SECTION=201.html

18 Shao Z, Janse E, Visser K, Meyer AS. What do verbal fluency tasks measure? Predictors of verbal fluency performance in older adults. Front Psychol 2014;5:772

19 Benito-Cuadrado MM, Esteba-Castillo S, Böhm P, Cejudo-Bolívar J, Peña-Casanova J. Semantic verbal fluency of animals: a normative and predictive study in a Spanish population. J Clin Exp Neuropsychol 2002;24(8):1117-1122

20 Ostrosky-Solis F, Gutierrez AL, Flores MR, Ardila A. Same or different? Semantic verbal fluency across Spanish-speakers from different countries. Arch Clin Neuropsychol 2007;22(3):367-377

21 Dell GS. A spreading-activation theory of retrieval in sentence production. Psychol Rev 1986;93(3):283-321

22 Luo L, Luk G, Bialystok E. Effect of language proficiency and executive control on verbal fluency performance in bilinguals. Cognition 2010;114(1):29-41

23 Roca M, Manes F, Chade A, et al. The relationship between executive functions and fluid intelligence in Parkinson's disease. Psychol Med 2012;42(11):2445-2452

24 Cutting LE, Materek A, Cole CA, Levine TM, Mahone EM. Effects of fluency, oral language, and executive function on reading comprehension performance. Ann Dyslexia 2009;59(1):34-54 